\title{
Oral health behavior and HbA1c in Indian adults with type 2 diabetes
}

\author{
Ashish Aggarwal and Sunil R. Panat \\ Department of Oral Medicine and Radiology, Institute of Dental Sciences, Bareilly, UP, India
}

(Received 20 June and accepted 18 September 2012)

\begin{abstract}
Prevention and treatment of oral diseases and diabetes require persistent daily self-care, as there is a mutual association between periodontitis severity and level of diabetes control. In this questionnaire study, we investigated oral health behavior, attitudes, and knowledge of diabetesrelated factors among 500 Indian adults with type 2 diabetes mellitus. The questionnaire asked about oral self-care, dental visits, self-perceived problems, and knowledge of the relationship between diabetes and oral health. The most recent glycosylated hemoglobin (HbA1c) value was obtained from patient medical records. Overall, $22 \%$ of participants reported twicedaily toothbrushing; women were more likely than men to brush twice daily $(P<0.001)$. With respect to age and diabetes control, participants aged 35-44 years with good diabetes control had the highest rate of twice-daily brushing $(P<0.001)$. Oral self-care and use of dental services were poor among participants. The present results indicate that Indians with type 2 diabetes need further promotion of oral self-care and regular dental checkups to compensate for their increased risk of oral disease. (J Oral Sci 54, 293-301, 2012)
\end{abstract}

Keywords: diabetes mellitus; oral self care; periodontal disease; dental services.

\section{Introduction}

Diabetes mellitus is a major global health problem,

Correspondence to Dr. Ashish Aggarwal, Department of Oral Medicine and Radiology, Institute of Dental Sciences, Pilibhit Bypass Road, Bareilly, Uttar Pradesh, India

Tel: +91-9358353566

E-mail: drashishagg@rediffmail.com and its prevalence is increasing worldwide (1). The number of estimated cases of diabetes increased from 30 million in 1985 to 135 million in 1995 and is projected to increase to 366 million by the year 2030 (2). Diabetes and its acute complications have become a significant health hazard worldwide, especially in the Indian subcontinent. According to the World Diabetes Atlas, India is projected to have around 51 million people with diabetes (3). With its rising incidence, diabetes has captured the attention of specialists and general practitioners in both medicine and dentistry.

Prevention and treatment of oral diseases and diabetes require persistent daily self-care (4). Oral self-care, which is part of general health self-care, comprises a wide spectrum of activities ranging from self-treatment, prevention, and diagnosis to seeking lay or professional care (5). Among dentate adults, regular dental visits are an opportunity for professional assistance in prevention, early detection, and treatment of periodontal diseases (6). Certain groups of individuals, such as people with diabetes, have a high risk for developing these diseases $(7,8)$. Periodontal disease has been called the sixth complication of diabetes (9). Because of these relationships, prevention and care of periodontitis - which involve consistent daily oral care at home and regular dental visits-are particularly important for diabetic patients (10). People with poor control of diabetes also have relatively high plaque levels (11). Many diabetic patients do not visit dental clinics regularly and miss more dental appointments than nondiabetic patients $(12,13)$.

Medical complications commonly associated with diabetes include renal disease, retinopathy, neuropathy, peripheral vascular disease, and coronary heart disease (14). Oral health complications reportedly associated with diabetes include tooth loss, gingivitis, periodontitis, 
and soft tissue pathologies (15). Periodontitis is perhaps the most widely noted oral manifestation of diabetes.

Individuals who do not maintain good oral hygiene are at higher risk for periodontal diseases. Consequently, this risk seems to be lower among people who control both their diabetes and oral health by maintaining comprehensive self-care (16). Regular professional dental cleaning may improve glycemic control, even among patients with poorly controlled diabetes (17).

Research by various authors indicates that improvement of oral health knowledge is necessary for implementation of proper oral self-care behaviors. Although knowledge of correct behaviors does not necessarily translate to practice (18), lack of essential oral health knowledge (e.g., ignorance of the warning signs and symptoms of oral disease) may increase the risks of diabetic oral complications and/or contribute to inadequate oral selfcare practices (19). In addition, misconceptions about oral health may actually lead to harmful behaviors (20) and could act as major barriers to efficient prevention and management of oral disease in diabetics.

Furthermore, accumulating evidence highlights the effect of oral disease on systemic ill health, including diabetes and heart disease. Thus, the attitude of diabetic patients to their oral health is important $(21,22)$. Previous studies indicate that oral health may not be a priority for diabetic patients and that these patients are less likely than nondiabetic patients to have visited a dentist (23). Furthermore, it was reported that twice-daily toothbrushing is less common in diabetic patients than in nondiabetic patients (5).

Despite worldwide recognition of the dangers of diabetes, the awareness and attitude of diabetic patients toward their heightened risk for oral diseases has not been fully addressed. To date, there are no data on oral health behavior, oral hygiene, and HbAlc level among Indians with type 2 diabetes. In addition, few studies have investigated oral self-care and use of dental services by diabetic patients. Thus, we investigated oral health attitudes, knowledge, and behavior with regard to diabetes-related factors among adults with type 2 diabetes. We also assessed their attitudes toward sustaining good oral health through proper oral hygiene and regular dental check-ups.

\section{Materials and Methods}

The study participants were 500 consecutive patients with type 2 diabetes who sought treatment at the Department of Oral Medicine and Radiology, Institute of Dental Sciences, Bareilly, Uttar Pradesh, India from September 1, 2010 to December 30, 2011. The criteria for inclusion were age $\geq 35$ years and presence of $\geq 1$ tooth in the oral cavity. Information on diabetes type and most recent glycosylated hemoglobin level (HbA1c) were obtained from patient medical records. Diabetes control was considered to be good if $\mathrm{HbA} 1 \mathrm{c}$ was $\leq 6.0 \%$, moderate if it was $6.1-7.5 \%$, and poor if it was $>7.5 \%$ (24). Informed, written consent was received from all patients who agreed to participate in the study. The participants completed a self-report questionnaire as they awaited their appointment at the outpatient clinic. The participants received oral and written instructions on questionnaire completion, and assurances of confidentiality. Completed questionnaires were collected from the participants as they left the clinic. Patients with intellectual impairments or reading difficulties were excluded. The study was approved by the Ethical Committee of the Institute of Dental Sciences, Bareilly, Uttar Pradesh, India.

\section{Questionnaire}

A self-administered questionnaire was used to assess the main outcomes of the study. Questionnaire items were carefully selected from relevant published reports in international journals $(2,5,6,15,24)$. The questions were closed-ended, and most were multiple-choice items with alternative statements. The questionnaire asked about 1) demographic characteristics - age, sex, education level, and habits like smoking and drinking, 2) medical history - type and duration of diabetes, HbAlc level, and diabetes complications, 3 ) oral hygiene behavior - use of toothbrush, fluoridated toothpaste, floss, mouthwash, and interdental cleaning aids, 4) utilization of professional dental services - most recent dental visit and reason, $5)$ knowledge of relationship between diabetes and oral health, and 6) self-perceived problems - dry mouth, altered taste sensation, caries, periodontitis, and missing teeth, among others.

Before the present study, we conducted a pilot study of 25 diabetic volunteers to assess participant responses to the questionnaire. None of the present patients refused to answer any questionnaire item.

\section{Statistical analysis}

The data were entered on a Microsoft Excel spreadsheet and imported into Statistical Package for Social Sciences (SPSS) version 17 (SPSS Inc., Chicago, IL, USA) for statistical analysis. Frequency distribution tables were produced, and the chi square test was used to assess associations of variables with level of diabetes control. A $P$-value less than 0.05 was considered statistically significant. 
Table 1 Characteristics of participants

\begin{tabular}{|c|c|c|}
\hline & Number & $\%$ \\
\hline \multicolumn{3}{|l|}{ Sex } \\
\hline Male & 266 & 53.2 \\
\hline Female & 234 & 46.8 \\
\hline \multicolumn{3}{|l|}{ Age group (y) } \\
\hline $35-44$ & 118 & 23.6 \\
\hline $45-54$ & 146 & 29.2 \\
\hline $55-64$ & 110 & 22 \\
\hline $65-75$ & 77 & 15.4 \\
\hline$>75$ & 49 & 9.8 \\
\hline \multicolumn{3}{|l|}{ Level of education } \\
\hline Low (elementary school) & 162 & 32.4 \\
\hline Medium (college degree) & 241 & 48.2 \\
\hline High (postgraduate study) & 97 & 19.4 \\
\hline \multicolumn{3}{|l|}{ Duration of disease (y) } \\
\hline$<2$ & 116 & 23.2 \\
\hline $2-5$ & 171 & 34 \\
\hline $6-10$ & 128 & 25.6 \\
\hline$>10$ & 85 & 17 \\
\hline \multicolumn{3}{|l|}{ Diabetes control (HbA1c) } \\
\hline$<6.0$ (good) & 279 & 55.8 \\
\hline $6.1-7.5$ (moderate) & 141 & 28.2 \\
\hline$>7.5$ (poor) & 80 & 16.0 \\
\hline \multicolumn{3}{|l|}{ Complications } \\
\hline Yes & 208 & 41.6 \\
\hline No & 292 & 58.4 \\
\hline \multicolumn{3}{|l|}{ Smoking } \\
\hline Nonsmoker & 291 & 58.2 \\
\hline Smoker & 140 & 28.0 \\
\hline Ex-smoker & 69 & 13.8 \\
\hline \multicolumn{3}{|l|}{ Alcohol use } \\
\hline Nondrinker & 257 & 51.4 \\
\hline Drinker & 243 & 48.6 \\
\hline$<7$ oz/week & 182 & 36.3 \\
\hline$>7 \mathrm{oz} /$ week & 61 & 12.3 \\
\hline
\end{tabular}

\section{Results}

A total of 500 patients were included in the study: $266(53.2 \%)$ men and $234(46.8 \%)$ women. The age range of patients was 35-87 years. When participants were subdivided into five age groups, $23.6 \%$ were aged $35-44,29.2 \%$ were $45-54,22 \%$ were $55-64,15.4 \%$ were $65-74$, and $9.8 \%$ were $\geq 75$. Regarding education, $32.4 \%$, $48.2 \%$, and $19.4 \%$ of patients had a low, medium, and high level of education, respectively. The most frequent (34\%) duration of diabetes was 2-5 years: $25.6 \%, 23.2 \%$, and $17 \%$ of patients had a duration of diabetes of 6-10 years, $<2$ years, and $>10$ years, respectively. Regarding glycemic control (HbA1c), 55.8\%, 28.2\%, and 16\%
Table 2 Oral self-care and use of dental services by participants

\begin{tabular}{|c|c|c|}
\hline \multicolumn{3}{|l|}{ Oral self-care among participants } \\
\hline \multicolumn{3}{|l|}{ Toothbrushing frequency (times per day) } \\
\hline$<1$ & 88 & $17.6 \%$ \\
\hline 1 & 245 & $49.0 \%$ \\
\hline 2 & 110 & $22.0 \%$ \\
\hline$>2$ & 57 & $11.4 \%$ \\
\hline \multicolumn{3}{|l|}{ Use of fluoridated toothpaste } \\
\hline Yes & 462 & $92.4 \%$ \\
\hline No & 38 & $7.6 \%$ \\
\hline \multicolumn{3}{|l|}{ Use of mouthwash } \\
\hline Yes & 92 & $18.4 \%$ \\
\hline No & 408 & $81.6 \%$ \\
\hline \multicolumn{3}{|l|}{ Interdental cleaning aids } \\
\hline No & 210 & $42 \%$ \\
\hline Yes & 290 & $58 \%$ \\
\hline Toothpick & 170 & $34 \%$ \\
\hline Dental floss & 81 & $16 \%$ \\
\hline Interdental Brush & 39 & $8 \%$ \\
\hline \multicolumn{3}{|l|}{ Frequency (times per day) } \\
\hline$<1$ & 32 & $11 \%$ \\
\hline 1 & 151 & $52 \%$ \\
\hline 2 & 66 & $23 \%$ \\
\hline$>2$ & 41 & $14 \%$ \\
\hline \multicolumn{3}{|l|}{ Professional dental care } \\
\hline \multicolumn{3}{|l|}{ Most recent dental visit } \\
\hline$<6$ months before & 268 & $53.6 \%$ \\
\hline 6-12 months before & 110 & $22.0 \%$ \\
\hline $1-2$ years before & 76 & $15.2 \%$ \\
\hline$>2$ years before & 46 & $9.2 \%$ \\
\hline \multicolumn{3}{|l|}{ Reason for last visit } \\
\hline Check-up & 54 & $10.8 \%$ \\
\hline Pain & 165 & $33.0 \%$ \\
\hline Cleaning & 62 & $12.4 \%$ \\
\hline Extraction & 107 & $21.4 \%$ \\
\hline Periodontal problem & 80 & $16.0 \%$ \\
\hline Other & 32 & $6.4 \%$ \\
\hline \multicolumn{3}{|l|}{ Reason for no dental visit during previous 2 years } \\
\hline a. Dental care is unpleasant & 92 & $18.4 \%$ \\
\hline b. Dental care is too expensive & 77 & $15.4 \%$ \\
\hline c. I didn't have a dental problem & 122 & $24.3 \%$ \\
\hline d. Could not make appointment due to work & 61 & $12.2 \%$ \\
\hline e. Did not know that I needed a dental check-up & 148 & $29.7 \%$ \\
\hline
\end{tabular}

of patients had good, moderate, and poor control, respectively, and $58.4 \%$ of patients had no diabetes complications according to their medical records at the time of their dental visit (Table 1).

Regarding frequency of brushing, $49 \%$ reported brushing once daily, as compared with $22 \%$ that brushed twice daily, $11.4 \%$ that brushed more than twice daily, 
Table 3 Self-perceived problems and knowledge of relationship between diabetes and oral health

\begin{tabular}{lrr}
\hline Self-perceived problems & & \\
Dry mouth & 195 & $39 \%$ \\
Altered oral sensation & 86 & $17.2 \%$ \\
Periodontitis & 63 & $12.6 \%$ \\
Caries & 57 & $11.5 \%$ \\
Missing teeth & 52 & $10.3 \%$ \\
Others & 47 & $9.4 \%$ \\
Relationship between diabetes and oral health & & \\
Does diabetes affect oral health? & \\
Yes & 192 & $38.4 \%$ \\
No & 308 & $61.6 \%$ \\
Does your dentist know about your oral health? & & \\
Yes & 236 & $47.2 \%$ \\
No & 264 & $52.8 \%$ \\
Referral from physician & & \\
Yes & 103 & $20.6 \%$ \\
No & 397 & $79.4 \%$ \\
\hline
\end{tabular}

and $17.6 \%$ that brushed less than once daily. Among these patients, $92.4 \%$ were using fluoridated toothpaste (Table 2).

Among patients with good or moderate control of diabetes, women were significantly more likely than men to brush twice daily $(P<0.001)$. In the age group 35-44 years, the proportion of individuals brushing twice daily was highest (67.1\%) among diabetics with good control $(P<0.001)$, whereas in the age group 45-54 years, the proportion of individuals brushing twice daily was highest (57.1\%) among diabetics with moderate control $(P<0.001)$. Patients with a high education level were significantly more likely to brush twice daily than those with a moderate or low level of education $(P<0.001)$. Patients with a diabetes duration of 2-5 years and good diabetes control had a higher rate of twice-daily brushing $(62.9 \%)$ than any other group $(P<0.001)$. Patients without diabetes complications had a higher rate of

Table 4 Rate (\%) of twice-daily toothbrushing according to level of diabetes control

\begin{tabular}{|c|c|c|c|c|c|c|}
\hline & \multicolumn{6}{|c|}{ Diabetes control } \\
\hline & \multicolumn{2}{|c|}{ Good } & \multicolumn{2}{|c|}{ Moderate } & \multicolumn{2}{|c|}{ Poor } \\
\hline & & $\%$ & & $\%$ & & $\%$ \\
\hline \multicolumn{7}{|l|}{ Sex } \\
\hline Male & 149 & 33.6 & 67 & 10.4 & 50 & 10 \\
\hline Female & 130 & 56.2 & 74 & 33.8 & 30 & 23.3 \\
\hline$P$ value & \multicolumn{2}{|c|}{$<0.001^{*}$} & \multicolumn{2}{|c|}{$0.001^{*}$} & \multicolumn{2}{|c|}{$0.106^{\mathrm{NS}}$} \\
\hline \multicolumn{7}{|l|}{ Age (y) } \\
\hline $35-44$ & 79 & 67.1 & 26 & 34.6 & 13 & 7.7 \\
\hline $45-54$ & 90 & 45.6 & 35 & 57.1 & 21 & 33.3 \\
\hline $55-64$ & 60 & 45 & 35 & 5.7 & 15 & 13.3 \\
\hline $65-75$ & 35 & 5.7 & 23 & 0 & 19 & 0 \\
\hline$>75$ & 15 & 0 & 22 & 4.5 & 12 & 16.7 \\
\hline$P$ value & \multicolumn{2}{|c|}{$<0.001^{*}$} & \multicolumn{2}{|c|}{$<0.001^{*}$} & \multicolumn{2}{|c|}{$0.052^{\mathrm{NS}}$} \\
\hline \multicolumn{7}{|c|}{ Level of education } \\
\hline Low & 60 & 18.3 & 63 & 11.1 & 39 & 5.1 \\
\hline Medium & 157 & 33.8 & 52 & 11.5 & 32 & 12.5 \\
\hline High & 62 & 95.2 & 26 & 73.1 & 9 & 66.7 \\
\hline$P$ value & \multicolumn{2}{|c|}{$<0.001^{*}$} & \multicolumn{2}{|c|}{$<0.001^{*}$} & \multicolumn{2}{|c|}{$<0.001^{*}$} \\
\hline \multicolumn{7}{|c|}{ Duration of disease (y) } \\
\hline$<2$ & 60 & 46.7 & 40 & 35 & 16 & 6.3 \\
\hline $2-5$ & 105 & 62.9 & 37 & 18.9 & 29 & 24.1 \\
\hline $6-10$ & 69 & 34.8 & 42 & 19 & 17 & 23.5 \\
\hline$>10$ & 45 & 11.1 & 22 & 13.6 & 18 & 0 \\
\hline$P$ value & \multicolumn{2}{|c|}{$<0.001^{*}$} & \multicolumn{2}{|c|}{$0.165^{\mathrm{NS}}$} & \multicolumn{2}{|c|}{$0.072^{\mathrm{NS}}$} \\
\hline \multicolumn{7}{|c|}{ Complications } \\
\hline Yes & 75 & 0 & 82 & 1.2 & 51 & 0 \\
\hline No & 204 & 60.3 & 59 & 52.5 & 29 & 41.4 \\
\hline$P$ value & \multicolumn{2}{|c|}{$<0.001 *$} & \multicolumn{2}{|c|}{$<0.001 *$} & \multicolumn{2}{|c|}{$<0.001 *$} \\
\hline
\end{tabular}

*: Statistically significant, NS: Not significant 
Table 5 Proportions of participants reporting a dental visit within the past 12 months according to level of diabetes control

\begin{tabular}{|c|c|c|c|c|c|c|}
\hline & \multicolumn{6}{|c|}{ Diabetes control } \\
\hline & \multicolumn{2}{|c|}{ Good } & \multicolumn{2}{|c|}{ Moderate } & \multicolumn{2}{|c|}{ Poor } \\
\hline & & $\%$ & & $\%$ & & $\%$ \\
\hline \multicolumn{7}{|l|}{ Sex } \\
\hline Male & 149 & 93.3 & 67 & 62.7 & 50 & 22 \\
\hline Female & 130 & 96.2 & 74 & 67.6 & 30 & 36.7 \\
\hline$P$ value & \multicolumn{2}{|c|}{$0.290^{\mathrm{NS}}$} & \multicolumn{2}{|c|}{$0.543^{\mathrm{NS}}$} & \multicolumn{2}{|c|}{$0.155^{\mathrm{NS}}$} \\
\hline \multicolumn{7}{|l|}{ Age (y) } \\
\hline $35-44$ & 79 & 100 & 26 & 100 & 13 & 15.4 \\
\hline $45-54$ & 90 & 94.4 & 35 & 77.1 & 21 & 42.9 \\
\hline $55-64$ & 60 & 91.7 & 35 & 51.4 & 15 & 46.7 \\
\hline $65-75$ & 35 & 91.4 & 23 & 4.3 & 19 & 5.3 \\
\hline$>75$ & 15 & 86.7 & 22 & 90.9 & 12 & 25 \\
\hline$P$ value & \multicolumn{2}{|c|}{$0.088^{\mathrm{NS}}$} & \multicolumn{2}{|c|}{$<0.001^{*}$} & \multicolumn{2}{|c|}{$0.027 *$} \\
\hline \multicolumn{7}{|c|}{ Level of education } \\
\hline Low & 60 & 93.3 & 63 & 52.4 & 39 & 30.8 \\
\hline Medium & 157 & 94.3 & 52 & 63.5 & 32 & 18.8 \\
\hline High & 62 & 96.8 & 26 & 100 & 9 & 44.4 \\
\hline$P$ value & \multicolumn{2}{|c|}{$0.671^{\mathrm{NS}}$} & \multicolumn{2}{|c|}{$<0.001^{*}$} & \multicolumn{2}{|c|}{$0.255^{\mathrm{NS}}$} \\
\hline \multicolumn{7}{|c|}{ Disease duration (y) } \\
\hline$<2$ & 60 & 98.3 & 40 & 85 & 16 & 18.8 \\
\hline $2-5$ & 105 & 98.1 & 37 & 54.1 & 29 & 27.6 \\
\hline $6-10$ & 69 & 88.4 & 42 & 64.3 & 17 & 52.9 \\
\hline$>10$ & 45 & 91.1 & 22 & 50 & 18 & 11.1 \\
\hline$P$ value & \multicolumn{2}{|c|}{$0.015^{*}$} & \multicolumn{2}{|c|}{$0.011 *$} & \multicolumn{2}{|c|}{$0.036^{*}$} \\
\hline \multicolumn{7}{|c|}{ Complications } \\
\hline Yes & 75 & 90.7 & 82 & 48.8 & 51 & 5.9 \\
\hline No & 204 & 96.1 & 59 & 88.1 & 29 & 65.5 \\
\hline$P$ value & \multicolumn{2}{|c|}{$0.076^{\mathrm{NS}}$} & \multicolumn{2}{|c|}{$<0.001^{*}$} & \multicolumn{2}{|c|}{$<0.001 *$} \\
\hline
\end{tabular}

twice-daily brushing than did those with complications $(P<0.001)$ (Table 4).

Mouthwash use was reported by $18.4 \%$ of patients. The most common device for interdental cleaning was toothpicks $(34 \%)$, followed by dental floss $(16 \%)$ and interdental brushes $(8 \%)$. Interdental cleaning aids were used at least once daily by $52 \%$ of patients; $42 \%$ of participants reported no interdental cleaning (Table 2).

Regarding the most recent dental visit, $53.6 \%$ of patients reported a visit within the past 6 months, $22 \%$ had a visit 6-12 months before, $15.2 \%$ had a visit $1-2$ years before, and $9.2 \%$ had a visit more than 2 years before. When level of diabetes control was assessed in relation to the proportion of patients reporting a dental visit within the past 12 months, there was no statistically significant difference between men and women. Intergroup comparison showed that patients aged 35-44 years with moderate diabetes control $(P<0.001)$ and those aged 45-54 years with poor diabetes control were most likely to have had a dental visit during the past 12 months $(P<0.027)$. Patients with a high level of education and moderate diabetes control were most likely to have had a dental visit during the past 12 months $(P<$ $0.001)$. The proportions of patients reporting a dental visit within the past 12 months significantly differed in relation to diabetes duration in the good, moderate, and poor control groups $(P<0.015, P<0.011$, and $P<0.036$, respectively). Patients with moderate control and no diabetes complications had a significantly higher rate of dental visits in the past 12 months than did patients with diabetes complications $(P<0.001)$ (Table 5).

The most common reason for the most recent dental visit was pain (33\%), followed by extraction (21.4\%), periodontal problem $(16 \%)$, and cleaning of teeth (12.4\%). Only $10.8 \%$ of patients reported visiting a dentist for a regular dental check-up. The main reason 
for not visiting a dentist for $>2$ years was unawareness of the need for a dental check-up (29.7\%) (Table 2).

The most frequently reported self-perceived problem was dry mouth (39\%), followed by altered oral sensation $(17.2 \%)$, periodontitis $(12.6 \%)$, caries $(11.5 \%)$, missing teeth $(10.3 \%)$, and other problems $(9.4 \%)$ (Table 3$)$.

Only $38.4 \%$ patients were aware that diabetes affects oral health. Most patients (52.8\%) reported that their dentist did not know they had diabetes. A large majority of patients (79.4\%) reported that they had never received a physician referral for dental care (Table 3).

\section{Discussion}

Oral diseases are increasingly regarded as behavioral illnesses (25). Adoption of healthy habits, including sufficient oral self-care and regular dental visits, is essential to control such diseases $(26,27)$. Prevention and treatment of oral diseases and diabetes require persistent daily self-care (4).

Maintenance of good oral hygiene was poor among the study participants. The proportion of those who brushed more than twice daily $(11.4 \%)$ was considerably lower than proportions reported by Karikoski et al. (38\%) and Murtomaa et al. (61\%) $(5,28)$. Even the proportion of participants who brushed once daily (49\%) was lower than those reported by Karikoski et al. (83\%) and Murtomaa et al. $(91 \%)(5,28)$. In a study of Finnish adults with diabetes, twice-daily brushing was reported by $50 \%$ of participants (12). The relatively small proportion of the present participants who brushed more than once daily is noteworthy in light of the Indian Dental Association (IDA) recommendation of twice-daily brushing.

The rate of twice-daily brushing was highest among patients with good (age group 35-44 years) and moderate diabetes control (age group 45-54 years). The rate of twice-daily brushing was lowest among those with poor control, which is in accordance with the results of Bakhshandeh et al. (6). The association between poor diabetes control and infrequent toothbrushing suggests that dental health education is important, especially in diabetic patients with poor control (10). Therefore, it seems particularly important to enhance toothbrushing self-efficacy in this population, which, in turn, essentially depends on compliance with diabetes self-care practices (29).

In our study, $92.4 \%$ of patients reported using fluoridated toothpaste. Such a higher rate of the response may be due to the fact that the present study was conducted in an urban setting, where most patients were aware of the advantages of using toothpaste while brushing.

With regard to situational factors, female sex and a high level of education were strong predictors of brushing frequency, as was the case in another study of dental health behavior (30). In the present study, $58 \%$ of patients reported using interdental cleaning aids, primarily toothpicks (34\%) and dental floss (16\%), which agrees with the findings of Bakhshandeh et al. (6). Because toothbrushing, whether done manually or with an electric toothbrush, cannot reach proximal and interdental areas, any program intended to reduce caries and periodontal disease must focus on thorough cleaning of those areas (26). Also, lack of information and financial difficulties might contribute to nonadherence with oral hygiene regimens. Diabetic patients should be encouraged to control gingivitis by flossing and to establish healthy habits to prevent periodontitis. Relatively simple interventions may be effective, particularly when proven techniques of behavioral change are used, such as supporting the development of personal behavioral intentions as described by Syrjälä et al. and Sniehotta et al. (10,31). The present results indicate that interdental cleaning is particularly important among Indians with diabetes.

In the present study, $28 \%$ of patients had a smoking habit, and $48.6 \%$ regularly consumed alcohol. There was no significant difference in oral hygiene practices between smokers and nonsmokers. In the study by Bakhshandeh et al., twice-daily brushing was more frequent among smokers than among nonsmokers (6). Cigarette smoking is an established risk factor for periodontal disease in healthy and diabetic patients and can be prevented through oral health promotion programs $(23,32)$. Although alcohol consumption is not considered a risk factor for periodontal disease, it can reduce the capacity of the liver to release glucose into the bloodstream and could cause hypoglycemia (33). Thus, diabetics should be made aware of the health hazards of smoking and alcohol consumption and encouraged to act accordingly.

Periodontitis treatment requires regular professional care and maintenance visits, including sub gingival plaque removal. In our study, only $53.6 \%$ and $22 \%$ of patients reported a dental visit within the previous 6 months and previous 6-12 months, respectively, which was higher than the rate in the study done by Murtomaa et al. (53\%) (28). It seems that the present patients were never informed of the importance of regular dental examinations. In additions, patients with diabetes complications and poor diabetes control had the lowest rate of dental visits. A dental visit within the previous 12 months was more frequently reported by patients who brushed twice daily, which conforms with the findings of Spangler and Konen. They noted that people with type 2 diabetes and good oral hygiene habits were more 
likely to use dental services annually (34). This implies that dental personnel can improve oral health behavior by both specific interventions and verbal persuasion, including encouragement and positive feedback $(35,36)$.

Unfortunately, $89.2 \%$ of the present patients visited their dentist only when they had a problem, which agrees with the findings of other authors (37), who found that patients with irregular dental visits had more plaque and calculus, more bleeding sites, and deeper mean probing depths. Thus, good plaque control and regular maintenance visits are critical in preventing and treating periodontal disease. Only $10.8 \%$ of our patients visited dentists for a regular dental check-up, which is much lower than the proportion reported by Karikoski et al. (47\%) (5). In our study, ignorance of the need for regular dental check-ups was the most common reason for not having received dental treatment within the past 2 years. Sakki et al. concluded that the attitude that dental care is unnecessary is a considerable obstacle to improving oral health (30).

Oral dryness is often unpleasant and painful and can have a negative effect on general quality of life. Oral dryness was a common complaint in the present population, and was reported by $39 \%$ of participants. A substantial reduction of salivary flow leading to xerostomia is the most common oral manifestation of diabetes. It is essential to inform diabetics of the beneficial properties of saliva. Washing and cleansing of the oral cavity prevents accumulation of plaque and debris, which can contribute to the increased risk of periodontal disease and dental caries among diabetics (38). Moreover, diabetics should be informed of the importance for keeping the oral cavity moist by stimulating salivary flow and frequent water drinking.

Another important finding in the present study is that a large majority of the participants had never been informed of the association between diabetes and oral health: almost $61 \%$ believed that diabetes had no influence on their oral health. Only a few participants reported believing that their diabetes had affected their periodontal status and dry mouth. In addition, 52.8\% of participants reported that their dentist did not know of their diabetes diagnosis. This may be due to inadequate history taking or patient reluctance to disclose a diabetes diagnosis. Perhaps patients believed that dental professionals did not need to know about their diabetes status, which is plausible, as most patients believed that there was no association between diabetes and oral health.

Our findings show that a subset of the participants had a higher risk of receiving inadequate dental treatment, due to their dental care habits and attitudes toward dental care. One explanation for this finding is that diabetes and its treatment necessitate regular, daily physical and dietary habits, control of blood and urinary glucose, and frequent visits to diabetes clinics. These requirements might make regular dental care difficult. Moreover, even when adequately treated, diabetes can lead to severe complications such as cardiovascular disease and retinopathy. This, too, might influence attitudes toward dental care among diabetics (12).

The present study had some limitations. No objective clinical data were collected to support the results obtained from the self-report questionnaire. Some bias may have been present due to the recruitment of diabetic patients from a dental outpatient clinic, and the results might therefore not be representative of the general diabetic population, although we acknowledge that this study is a convenience sample. The use of self-reported data also limits our ability to validate the present findings. Response bias may have influenced individuals to overreport dental visits and brushing and flossing behaviors.

It is essential for dental professionals to raise awareness among diabetic patients of their increased risk for oral diseases and the impact of oral health on their general health. Early detection and treatment of tooth caries, periodontal disease, and other oral diseases would be of enormous benefit in protecting diabetic patients from the harmful oral complications associated with diabetes. Furthermore, dental professionals and the relevant government agencies should promote awareness of the relationship between diabetes and oral heath in order to prevent harmful dental complications and expensive treatment.

Dental services are covered by both the private and public sectors in India. Adults with diabetes are entitled to state-subsidized public dental care, which depends on the health care resources of the municipality. Our results indicate that these benefits are not fully utilized. Dental practitioners should play an educational role, both in clinical practice and at an organizational level, with closer integration between dental and diabetes professions, so at to raise awareness of oral health issues in the diabetic population.

In conclusion, Indians with type 2 diabetes reported suboptimal oral hygiene behavior. Our findings suggest that there is a significant need for increased knowledge of periodontal disease and adoption of preventive oral hygiene behaviors that would improve oral health among diabetics. Furthermore, because some people with diabetes do not seek regular dental care, all health care professionals should be encouraged to support efforts for more-comprehensive oral health, an integral 
part of general health. Thus, improvement in oral health behavior among diabetics is essential to compensate for their higher risk of oral diseases.

\section{References}

1. Tanwir F, Altamash M, Gustafsson A (2009) Effect of diabetes on periodontal status of a population with poor oral health. Acta Odontol Scand 67, 129-133.

2. Eldarrat AH (2011) Diabetic patients: their knowledge and perception of oral health. Libyan J Med 6, doi: 10.3402/ljm.v6i0.5691.

3. Anjana RM, Ali MK, Pradeepa R, Deepa M, Datta M, Unnikrishnan R, Rema M, Mohan V (2011) The need for obtaining accurate nationwide estimates of diabetes prevalence in India - rationale for a national study on diabetes. Indian J Med Res 133, 369-380.

4. Kneckt MC, Syrjälä AM, Laukkanen P, Knuuttila ML (1999) Self-efficacy as a common variable in oral health behavior and diabetes adherence. Eur J Oral Sci 107, 89-96.

5. Karikoski A, Ilanne-Parikka P, Murtomaa H (2002) Oral self-care among adults with diabetes in Finland. Community Dent Oral Epidemiol 30, 216-223.

6. Bakhshandeh S, Murtomaa H, Vehkalahti MM, Mofid R, Suomalainen K (2008) Oral self-care and use of dental services among adults with diabetes mellitus. Oral Health Prev Dent 6, 279-286.

7. Katz PP, Wirthlin MR, Szpunar SM, Selby JV, Sepe SJ, Showstack JA (1991) Epidemiology and prevention of periodontal disease in individuals with diabetes. Diabetes Care 14, 375-385.

8. Genco RJ (1996) Current view of risk factors for periodontal diseases. J Periodontol 67, 1041-1049.

9. Löe H (1993) Periodontal disease. The sixth complication of diabetes mellitus. Diabetes Care 16, 329-334.

10. Syrjälä AM, Kneckt MC, Knuuttila ML (1999) Dental self-efficacy as a determinant to oral health behaviour, oral hygiene and HbAlc level among diabetic patients. J Clin Periodontol 26, 616-621.

11. Karjalainen KM, Knuuttila ML, von Dickhoff KJ (1994) Association of the severity of periodontal disease with organ complications in type 1 diabetes patients. J Periodontol 65, 1067-1072.

12. Thorstensson H, Falk H, Hugoson A, Kuylenstierna J (1989) Dental care habits and knowledge of oral health in insulin-dependent diabetics. Scand J Dent Res 97, 207-215.
13. Pohjamo L, Tervonen T, Knuuttila M, Nurkkala $\mathrm{H}$ (1995) Adult diabetic and nondiabetic subjects as users of dental services. A longitudinal study. Acta Odontol Scand 53, 112-114.

14. Orchard TJ, Dorman JS, Maser RE, Becker DJ, Drash AL, Ellis D, LaPorte RE, Kuller LH (1990) Prevalance of complications in IDDM by sex and duration. Pittsburgh Epidemiology of Diabetes Complications Study II. Diabetes 39, 1116-1124.

15. Moore PA, Orchard T, Guggenheimer J, Weyant RJ (2000) Diabetes and oral health promotion: a survey of disease prevention behaviors. J Am Dent Assoc 131, 1333-1341.

16. Oliver RC, Tervonen T, Flynn DG, Keenan KM (1993) Enzyme activity in crevicular fluid in relation to metabolic control of diabetes and other periodontal risk factors. J Periodontol 64, 358-362.

17. Grossi SG, Skrepcinski FB, DeCaro T, Robertson DC, Ho AW, Dunford RG, Genco RJ (1997) Treatment of periodontal disease in diabetics reduces glycated hemoglobin. J Periodontol 68, 713-719.

18. Kay EJ, Locker D (1996) Is dental health education effective? A systematic review of current evidence. Community Dent Oral Epidemiol 24, 231-235.

19. Slaughter A, Smith VJ, Taylor L (2004) Progressing toward a more culturally competent approach to dental care for African American elders. Spec Care Dentist 24, 301-307.

20. Yuen HK, Wiegand RE, Slate EH, Magruder KM, Salinas CF, London SD (2008) Dental health knowledge in a group of Black adolescents living in rural South Carolina. J Allied Health 37, 15-21.

21. Montebugnoli L, Servidio D, Miaton RA, Prati C, Tricoci P, Melloni C, Melandri G (2005) Periodontal health improves systemic inflammatory and haemostatic status in subjects with coronary heart disease. J Clin Periodontol 32, 188-192.

22. Saremi A, Nelson RG, Tulloch-Reid M, Hanson RL, Sievers ML, Taylor GW, Shlossman M, Bennett PH, Genco R, Knowler WC (2005) Periodontal disease and mortality in type 2 diabetes. Diabetes Care 28, 27-32.

23. Tomar SL, Lester A (2000) Dental and other health care visits among U.S. adults with diabetes. Diabetes Care 23, 1505-1510.

24. Sandberg GE, Sundberg HE, Wikblad KF (2001) A controlled study of oral self-care and selfperceived oral health in type 2 diabetic patients. Acta Odontol Scand 59, 28-33.

25. Petersen PE, Bourgeois D, Bratthall D, Ogawa $H$ (2005) Oral health information systems - towards 
measuring progress in oral health promotion and disease prevention. Bull World Health Organ 83, 686-693.

26. Löe H (2000) Oral hygiene in the prevention of caries and periodontal disease. Int Dent J 50, 129-139.

27. Axelsson P, Albandar JM, Rams TE (2002) Prevention and control of periodontal diseases in developing and industrialized nations. Periodontol 2000 29, 235-246.

28. Murtomaa H, Metsäniitty M (1994) Trends in toothbrushing and utilization of dental services in Finland. Community Dent Oral Epiodemiol 22, 231-234.

29. Morris AD, Boyle DI, McMohan AD, Greene SA, MacDonald TM, Newton RW (1997) Adherence to insulin treatment, glycaemic control, and ketoacidosis in insulin-dependent diabetes mellitus. Lancet 350, 1505-1510.

30. Sakki TK, Knuuttila ML, Anttila SS (1998) Lifestyle, gender and occupational status as determinants of dental health behavior. J Clin Periodontol 25, 566-570.

31. Sniehotta FF, Araújo Soares V, Dombrowski SU (2007) Randomized controlled trial of a one-minute intervention changing oral self-care behavior. $\mathrm{J}$ Dent Res 86, 641-645.
32. Haber J, Kent RL (1992) Cigarette smoking in periodontal practice. J Periodontol 63, 100-106.

33. Franz MJ, Bantle JP, Beebe CA, Brunzell JD, Chiasson JL, Garg A, Holzmeister LA, Hoogwerf B, Mayer-Davis E, Mooradian AD, Purnell JQ, Wheeler M (2004) Nutrition principles and recommendations in diabetes. Diabetes Care 27, Suppl 1, 36-46.

34. Spangler JG, Konen JC (1994) Oral health behaviors in medical patients with diabetes mellitus. J Dent Hyg 68, 287-293.

35. Anderson RM, Funnell MM, Butler PM, Arnold MS, Fitzgerald JT, Feste CC (1995) Patient empowerment. Results of a randomized controlled trial. Diabetes Care 18, 943-949.

36. Wolfe GR, Stewart JM, Maeder LA, Hartz GW (1996) Use of Dental Coping Beliefs Scale to measure cognitive changes following oral hygiene interventions. Community Dent Oral Epidemiol 24, 37-41.

37. Greenstein G (2008) Practical periodontics: a review of core periodontal treatment principles. Dent Today 27, 70-73.

38. Finney LS, Finney MO, Gonzalez-Campoy JM (1997) What the mouth has to say about diabetes. Careful examinations can avert serious complications. Postgrad Med 102, 117-126. 